\title{
ASSESSMENT IMPACT OF USING LOCALLY PRODUCED PROBIOTIC BACTERIA ON THE PRODUCTIVE AND REPRODUCTIVE PERFORMANCE OF HOLSTEIN DAIRY COWS
}

\author{
EL-GARHI M.S. ${ }^{1}$; SOLTAN M.A. ${ }^{2}$; AHMED H.A. ${ }^{3}$; MERVAT A. ABDEL LATIF ${ }^{3}$; GALAL M. ${ }^{1}$ \\ and EL-BORDENY N.E. ${ }^{4}$ \\ ${ }^{1}$ Animal Reproduction Research Institute, Agricultural Research Center, Giza, Egypt. \\ ${ }^{2}$ Department of Nutrition and Veterinary Clinical Nutrition, Faculty of Veterinary Medicine, Alexandria University, Egypt \\ ${ }^{3}$ Department of Nutrition and Veterinary Clinical Nutrition, Faculty of Veterinary Medicine, Damanhour University, Egypt \\ ${ }^{4}$ Department of Animal Production, Faculty of Agric., Ain Shams Univ., P.O. Box 68 Hadayek Shoubra, 11241, Cairo, Egypt.
}

Received: 21 May 2019; Accepted: 2 July 2019

\begin{abstract}
The current work was conducted for assessment impact of using locally produced probiotic bacterial strains on the production and reproductive performance of Holstein dairy cows, those probiotic strains were isolated from saliva and fecal matter of suckling calves and rumen liquor of dairy cows. Thirty multiparous Holstein dairy cows at last 3 weeks of pregnancy were allotted to two groups (15 per each). $1^{\text {st }}$ group was the control group (C), fed transition diet (close up diet then post calving diet) without probiotic supplement, $2^{\text {nd }}$ group was the treated group (T), fed the same transition diets improved with $10^{6} \mathrm{CFU} / \mathrm{kg}$ DM feed of the produced probiotic supplement. Daily milk yield of each cow were recorded for 3 months postpartum (PP). Results revealed that cows in the treated group significantly $(\mathrm{P}<0.05)$ lost less BCS till day 30 PP than control then they gained more score at 3 months than control, probiotic supplementation non significantly $(\mathrm{P} \geq 0.05)$ increased average DMI throughout the whole experiment., probiotic supplementation improved $(\mathrm{P} \geq 0.05)$ average milk-to-feed ratio throughout the whole experiment. Treated group had a significant $(\mathrm{P}<0.05)$ increase in cholesterol and AST (Aspartate aminotransferase) levels than control. Milk yield non significantly $(\mathrm{P} \geq 0.05)$ increased during whole experimental period, moreover increased $(\mathrm{P} \geq 0.05)$ average milk production throughout the whole experimental period by about $7.3 \%$ compared to control, while probiotic supplementation non significantly ( $\mathrm{P} \geq 0.05)$ increased average milk fat $\%$ and yield (kg/day) or average milk protein $\%$ and yield, while had no clear effect on average solid not fat, milk lactose and ash percentages or yield compared to control. The effect of probiotics on reproduction revealed that cows in treated group had shorter days open, lesser I.N, fewer repeat breeders and better uterine and cervical involution and better conception rate at $3^{\text {rd }}$ insemination (CR3) than control. So it can be concluded that, adding the produced probiotics supplement to dairy cows ration improved cows transition period BCS, milk production, milk quality and had a positive effect on reproductive performance and postpartum uterine involution.
\end{abstract}

Key words: Probiotics bacteria, milk yield, milk quality and reproductive performance

\section{INTRODUCTION}

Dairy production is a challenging business. It is known that the main goal of dairying is producing milk, but a lot of factors can influence a cow's milk production (MP). These factors include: stressors from environment, housing environment, management practices, diet, cow genetics, and the health of the cow. Dairy managers use tools and management practices to minimize and/or prevent obstacles that may negatively affect milk production. One such tool is probiotic bacteria in the diet.

Corresponding author: Dr. EL-GARHI M.S. E-mail address: m_elgarhi2005@yahoo.com Present address: Animal Reproduction Research Institute, Agricultural Research Center, Giza, Egypt.
Fuller (1989) defined probiotics or direct feed microbial (DFM) as "preparations consisting of live micro-organisms or microbial stimulants which affect the endogenous microflora of the recipient. Adult dairy cattle are primarily fed probiotic bacteria from transition to mid lactation due to the stressors involved at those stages in production. There is in consistence between previous studies on its effect on milk production and milk constituents, Qiao et al. (2010) observed an increase in milk yield and milk protein for cows supplemented with Bacillus licheniformis, but not for Bacillus subtilis. Meanwhile Mongkolthanaruk (2012) reported Positive effects of Bacillus species as increased milk yield, fat-corrected milk, and milk protein. Kritas et al. (2006) and Qiao et al. (2010) reported an even 
greater increase in milk yield of 3.1 and $3.2 \mathrm{~kg} / \mathrm{d}$ with Bacillus subtilisnatto.

In similar ways, many authors reported that probiotic supplementation affected significantly on milk protein (\%) (Rossow et al., 2018; Hossain et al., 2014), milk lactose (\%) (Nocek and Kautz, 2006; Hossain et al., 2014) and SNF\% (Hossain et al., 2014). While, some authors reported no significant influence of probiotics on yield of milk protein \% (Weiss et al., 2008; Maamouri et al., 2014), lactose \% (Maamouri et al., 2014; Stella et al., 2007) and ash \% (Maamouri et al., 2014).

According to recent statistics the culling rate of dairy cows during 2013 reached $41.7 \%$ (Can West and Valacta, 2013). Among all the culling reasons, reproductive failure was rated as the number one, accounting for $15.4 \%$ of the total culling rate (Can West and Valacta, 2013). The occurrence of health problems during the transition period is clearly a major complexed factor for subsequent reproductive performance (Ferguson, 2001), resulting in additional economic losses, poor transitions, also result in milk income losses. It has been noticed that the DFM supplementation significantly improves the reproductive ability, conception rate and fertility (Abu ElElla and Kommonna, 2013) as well as the immune response (young, 2012).

Since the beneficial effects of probiotics are strain dependent, it has been suggested that combinations of different probiotic strains may be more effective than single strain probiotics (Timmerman et al., 2004). The rationale for multiple organisms comes from potential synergistic actions. It seems that probiotic strains of animal origin due to "host specific effect" are more effective (Fuller, 1989), and there are some studies that show animals generally benefits from probiotic microorganisms isolated from their own digestive tracts (Walter, 2005). There are limited researches in dairy cows fed multi-strains of probiotics from calves and dairy cattle origin, so, the purpose of this study was feeding probiotics (a laboratory produced probiotics), of calves and dairy cows origin, isolated from a farm to be used in the same farm, during the transition period of cows to evaluate its effect on cows' health, production and reproductive performance.

\section{MATERIALS AND METHODS}

This experiment was carried out at Sanad farm (private Holstein dairy farm in Damietta governorate - Egypt) during the period from September, 2016 to June 2017 to evaluate impact of using locally produced probiotic bacterial stains on the productive and reproductive performance of Holstein dairy cows.

Microbial strains and growth condition of the used probiotics: Fifteen probiotic isolates were isolated from calves saliva and feces and rumen liquor of dairy cows revealed on 3 bacterial strains characterized and identified to be propagated and used as a probiotic supplementation. Lactobacilli isolates were grown on MRS broth (Oxoid) while Streptococci isolates were grown on M17 broth (Difco), after that the broth media incubated for $24 \mathrm{~h}$ at $37{ }^{\circ} \mathrm{C}$. The strains were activated two or three times in order to obtain high biomasses in the stationary phase.

Genetic identification of isolated and tested strains: The isolates were identified according to 16sRNA in Sigma Company (Germany) by the automated sequencer (Table 1).

Table 1: The genetic identification of the tested probiotics stains.

\begin{tabular}{lllll}
\hline \multicolumn{3}{c}{ Probiotic strain } & Isolation source & Isolation media \\
\hline $\begin{array}{l}\text { Lactobacillus farraginis strain NRIC } \\
\text { ribosomal RNA gene, partial sequence, NR } 041467.1\end{array}$ & $\begin{array}{c}\text { Calves Saliva and Feces, } \\
\text { Rumen liquor }\end{array}$ & MRS- M17 \\
\hline $\begin{array}{l}\text { Lactobacillus } \\
\text { ribosomal RNA gene. Partial sequence, NR } 113820.1\end{array}$ & Calves saliva & M17 \\
\hline $\begin{array}{l}\text { Lactobacillus rhamnosus strain NBRC } \\
\text { ribosomal RNA gene, partial sequence, NR } 113332.1\end{array}$ & Calves Feces, rumen liquor & M17 \\
\hline
\end{tabular}

\section{Cows, experimental design and diets:}

Thirty multiparous Holstein dairy cows at last 3 weeks of pregnancy were allotted to two groups (15 per each). Total mixed rations (TMR) were formulated to meet the predicated requirements for energy, protein, minerals and vitamins during prepartum and postpartum periods according to NRC
(2001). Ingredient and chemical composition of the basal diets is presented in table (2). The first group was assigned to the control group without treatment, while the other group (treated group) fed on the basal diet with $10^{6} \mathrm{CFU} / \mathrm{kg} \mathrm{DM}$ feed of the produced probiotic supplement. 
Table 2: Ingredients (As-fed basis) and proximate analysis of the experimented rations (post calving diet).

\begin{tabular}{|c|c|c|}
\hline & $\begin{array}{c}\text { Post-calving diet } \\
\text { As- fed basis (kg/head /day) }\end{array}$ & $\begin{array}{c}\text { Pre-calving diet } \\
\text { As-fed basis (kg/head /day) }\end{array}$ \\
\hline \multicolumn{3}{|l|}{ Ingredients } \\
\hline Corn grain, ground, dry & 8 & 4.84 \\
\hline Soybean meal $47 \%$ & 3.5 & 2.33 \\
\hline Flax (Linseed whole lent) & 1.5 & 1 \\
\hline Corn silage & 20 & 25 \\
\hline Alfalfa hay & 5 & - \\
\hline Barseem (Egyptian clover) & 3.5 & - \\
\hline Sodium Bicarbonate & 0.24 & - \\
\hline Magnesium oxide & 0.04 & .03 \\
\hline Di- basic calcium phosphate & 0.04 & .06 \\
\hline Salt & 0.06 & - \\
\hline Calcium carbonate & 0.1 & - \\
\hline Premix $^{1}$ & 0.1 & .03 \\
\hline NEW T-NIL®Dry ${ }^{2}$ (Antimycotoxin) & 0.04 & 0.03 \\
\hline Total & 42.1 & 33.45 \\
\hline \multicolumn{3}{|l|}{ Chemical analysis: } \\
\hline $\mathrm{CP}(\% \text { of } \mathrm{DM})^{3}$ & 16.5 & 14.6 \\
\hline NEL (M. Cal. /kg. DM) ${ }^{4}$ & 1.65 & 2.81 \\
\hline $\mathrm{NDF}(\% \mathrm{DM})^{5}$ & 28.4 & 28.3 \\
\hline $\operatorname{ADF}(\% \quad \mathrm{DM})^{6}$ & 18.6 & 17 \\
\hline Forage NDF (\% DM) & 21.8 & 21.7 \\
\hline $\mathrm{TDN}(\% \text { of } \mathrm{DM})^{7}$ & 74 & 77 \\
\hline E.E $(\% \text { of DM })^{8}$ & 4.8 & 5.2 \\
\hline $\operatorname{RUP}(\% \text { of } \mathrm{CP})^{9}$ & 38.75 & \\
\hline
\end{tabular}

1= (Vit. A 10000000 IU, Vit D3 2500000 IU, Vit. E 35000 mg, Biotin 1000 mg, Zinc 100000 mg, Mn 80000 mg, Cu 30000 $\mathrm{mg}$, I $800 \mathrm{mg}$, Co $400 \mathrm{mg}$, Se $300 \mathrm{mg}$, Caco3 to $3 \mathrm{~kg}$ ).

$2=$ Sorbic acid $0.05 \%$, Citric acid $0.75 \%$, Calcium propionate $10.5 \%$, Copper sulphate $5 \%$, Inactivated yeast (Saccharomyces Cerevisiae) $2 \%$, Sapiolite $41.7 \%$, Bentonite $40 \%$

$3=$ crude protein, $4=$ net energy for lactation, $5=$ neutral detergent fiber, $6=$ acid detergent fiber, $7=$ Total digestible nutrients, $8=$ ether extract, $9=$ rumen undegredable protein

\section{Feed intake and Body condition score:}

Cows were fed a total mixed ration (where concentrates and roughages were mixed mechanically using mixer wagon in the farm), the two groups fed equal diets, cows were fed close up diet for three weeks till parturition then fed post calving ration till end of the experiment (12 weeks postpartum). Cows were fed the post calving diet in quantities to provide excess of the expected daily intake for ad libitum consumption with feed refusal (3-5\% of the total diet), while the Pre-calving cows were given a restricted diet from the close up ration and fed rice straw for free choice, cows were given a free access to water. Feed consumption was recorded daily by weighing feeds offered to and refused per each group.
Body condition scores of animals were performed by one evaluator using visual examination and palpation at the beginning of the experiment, 3 weeks before parturition then at day of parturition and at $4^{\text {th }}$ weeks PP and at $12^{\text {th }}$ weeks post calving, it was evaluated as described by Surinder et al. (1987).

\section{Sampling, measurements and analyses:}

Samples from the experimental ration were taken periodically for proximate analysis three times each every one month. Milk samples were taken one time every month for analysis of Milk constituents using milk scan. Milk samples were taken to the National Research Center for analysis of Milk constituents by 
mid infra-red spectroscopy with a milk scan 4000 (Biggs et al., 1987). Samples are taken from the same quarter before and after milking. Whole blood samples for progesterone concentration were collected in the mid luteal phase of $1^{\text {st }}$ and $2^{\text {nd }}$ insemination (7-12 day after ovulation) and at day 20 from $1^{\text {st }}$ and $2^{\text {nd }}$ insemination.

Also blood samples were collected 3 months PP at four hours after morning feeding at 8:00 am from the jugular vein to determine the biochemical parameters (blood metabolites) (Cholesterol, Triglyceride, ALT, AST, total protein and albumin) spectrophotometrically measured (T80 UV/VIS Spectrometer, PG Instruments Ltd, UK).

Collected Samples were centrifuged at $3000 \mathrm{xg}$ for 10 min. at $4^{\circ} \mathrm{C}$, to harvest serum, samples were frozen at $\left(-20^{\circ} \mathrm{C}\right)$ until required for analysis.

\section{Analytical procedures:}

Proximate analysis of experimental diets: Proximate analysis of dietary ingredients and experimental rations including dry matters (DM), crude protein $(\mathrm{CP})$, ether extract (EE), ash and nitrogen free extract (NFE) were analyzed according to A.O.A.C. (2000). Neutral detergent fiber (NDF) and acid detergent fiber (ADF), were analyzed by Ankom 2000 Fiber Analyzer as described by Van Soest et al. (1991).

Blood biochemistry and hormonal assay: Blood serum biochemistry was carried out at labs of Animal Nutrition, Animal Production Department, Faculty of Agriculture, Ain Shams University. Blood serum samples were analyzed using commercial kits. Total plasma protein concentrations was determined as described by Cannon et al. (1974), albumin concentrations was determined using method of Doumas et al. (1971), Triglycerides and cholesterol were determined according to Stein et al. (1987),
Alanine amino transferase (ALT) and aspartate amino transferase (AST) activities were calorimetrically determined according to AST and ALT kits based on reaction of Young (1990). Progesterone in was determined by using ELISA (Autrere and Benson., 1976).

Reproductive measures: Reproductive tract of all cows will be examined by using ultrasound scanning at 22-29 days post-partum and at 38-45 days postpartum for examination of uterine evolution (cervix diameter, two uterine horns diameters). Conception rate at $1^{\text {st }}, 2^{\text {nd }}$ and $3^{\text {rd }}$ service were calculated only for services accompanied by ovulation. Successful pregnancy will be defined by using ultrasound scanning at day 42 after mating. Insemination number per 1 st and 2 nd conception, percentage of repeat breeders, days interval from calving to 1 st estrus and 1 st insemination and days open will be measured. The detailed description of the reproductive performance evaluation is according to Westwood et al. (2000).

Statistical analysis: Obtained data were analyzed using the statistical package SPSS for Windows XP V15.0 (SPSS Inc., Chicago, USA, 2007). The significance of differences between treated samples was evaluated using Anova test.

\section{RESULTS}

\section{Body condition score (BCS):}

Bacterial probiotic supplementation of dairy cattle ration reduced $\mathrm{BCS}$ (table 3) at calving by about $11.9 \%$ while BCS of control group reduced by about $12.7 \%$ compared to BCS of the same group 3 weeks pre-partum. Moreover, bacterial probiotic supplementation reduced $\mathrm{BCS}$ at $4^{\text {th }}$ weeks postpartum by about $3.9 \%$ while BCS of control group reduced by about $14.5 \%$ compared to BCS of the same group at calving.

Table 3: Effect of dietary bacterial probiotic supplementation on body condition score (BCS) of dairy cows.

\begin{tabular}{ccc}
\hline \multirow{2}{*}{ Stage of lactation (weeks postpartum) } & \multicolumn{2}{c}{ Treatment } \\
\cline { 2 - 3 } & Control & Probiotic supplementation \\
\hline 3 weeks pre-partum & $4.03 \pm 0.059^{\mathrm{a}}$ & $3.52 \pm 0.063^{\mathrm{b}}$ \\
\hline At calving & $3.52 \pm 0.059^{\mathrm{a}}$ & $3.10 \pm 0.055^{\mathrm{b}}$ \\
\hline 4 weeks post-partum & $3.01 \pm 0.056^{\mathrm{a}}$ & $2.98 \pm 0.052^{\mathrm{a}}$ \\
\hline 12 weeks post-partum & $3.21 \pm 0.044^{\mathrm{a}}$ & $3.18 \pm 0.059^{\mathrm{a}}$ \\
\hline
\end{tabular}

Values are expressed as means \pm standard error (SE). Means within the same row of different litters are significantly different at $(\mathrm{P}<0.05)$.

\section{Dry Matter intake (DMI):}

No difference was observed by using bacterial probiotic in the lactating cows diet in dry matter intake (DMI) during first and $2^{\text {nd }}$ four weeks postpartum compared to control, as shown in table (4). However, probiotic supplementation significantly
$(\mathrm{P}<0.05)$ increased DMI during $3^{\text {rd }}$ month postpartum by about $6.9 \%$ and non-significantly $(\mathrm{P} \geq 0.05)$ increased average DMI throughout the whole experimental period by about $3.3 \%$ compared to control. 
Table 4: Effect of dietary bacterial probiotic supplementation on dry matter intake (DMI Kg/day/cow) of dairy cows.

Treatment

Stage of lactation (weeks postpartum)

\begin{tabular}{cc}
\hline Control & Probiotic supplementation \\
\hline $18.55 \pm 0.45^{\mathrm{a}}$ & $18.71 \pm 0.30^{\mathrm{a}}$ \\
\hline $20.17 \pm 0.54^{\mathrm{a}}$ & $20.43 \pm 0.71^{\mathrm{a}}$ \\
\hline $24.36 \pm 0.55^{\mathrm{b}}$ & $26.05 \pm 0.44^{\mathrm{a}}$ \\
\hline $21.03 \pm 0.47^{\mathrm{a}}$ & $21.73 \pm 0.70^{\mathrm{a}}$
\end{tabular}

Values are expressed as means \pm standard error (SE). Means within the same row of different litters are significantly different at $(\mathrm{P}<0.05)$.

\section{Milk production:}

The results of milk production is summarized in table 5. It was observed that probiotic supplementation in lactating cows ration non significantly $(\mathrm{P} \geq 0.05)$ increased milk yield during $1^{\text {st }}, 2^{\text {nd }}$ and $3^{\text {rd }}$ month postpartum by about $4.6 \%, 9.9 \%$ and $0.15 \%$ respectively, moreover increased $(\mathrm{P} \geq 0.05)$ average milk production throughout the whole experimental period by about $7.3 \%$ compared to control.

Table 5: Effect of dietary bacterial probiotic supplementation on postpartum milk production (Kg/day/cow) of dairy cows.

\begin{tabular}{ccc}
\hline & \multicolumn{2}{c}{ Treatment } \\
\cline { 2 - 3 } Stage of lactation (weeks postpartum) & Control & Probiotic supplementation \\
\cline { 2 - 3 } $0-4$ & $33.77 \pm 1.22^{\mathrm{a}}$ & $35.31 \pm 2.05^{\mathrm{a}}$ \\
\hline $5-8$ & $34.27 \pm 1.10^{\mathrm{a}}$ & $37.65 \pm 2.67^{\mathrm{a}}$ \\
\hline $9-12$ & $33.65 \pm 0.78^{\mathrm{a}}$ & $33.70 \pm 2.34^{\mathrm{a}}$ \\
\hline $0-12($ Average $)$ & $33.08 \pm 0.98^{\mathrm{a}}$ & $35.49 \pm 2.97^{\mathrm{a}}$ \\
\hline
\end{tabular}

Values are expressed as means \pm standard error (SE). Means within the same row of different litters are significantly different at $(\mathrm{P}<0.05)$.

\section{Milk composition:}

The effect of probiotic supplementation in dairy cattle ration on milk composition is presented in table (6). It was noticed that bacterial probiotic supplementation in dairy cows ration non significantly $(\mathrm{P} \geq 0.05)$ increased average milk fat $\%$ and yield ( $\mathrm{kg} /$ day) or average milk protein $\%$ and yield by about $(20.5 \%$ and $10.1 \%$ ) and $(1.5 \%$ and $3.7 \%)$ respectively, while had no clear effect on average solid not fat, milk lactose and ash percentages or yield compared to control. 
Table 6: Effect of dietary bacterial probiotic supplementation on postpartum milk composition of dairy cows.

\begin{tabular}{|c|c|c|c|c|c|}
\hline \multirow{2}{*}{ Items } & \multirow{2}{*}{ Treatment } & \multicolumn{4}{|c|}{ Stage of lactation (weeks postpartum) } \\
\hline & & $0-4$ & $5-8$ & $9-12$ & $0-12$ (average) \\
\hline \multicolumn{6}{|l|}{ Solid not fat (SNF) } \\
\hline \multirow{2}{*}{ SNF\% } & Control & $8.66 \pm 0.12^{\mathrm{b}}$ & $8.69 \pm 0.19^{\mathrm{a}}$ & $9.42 \pm 0.24^{\mathrm{a}}$ & $8.93 \pm 0.16^{\mathrm{a}}$ \\
\hline & Treated & $9.03 \pm 0.18^{\mathrm{a}}$ & $8.14 \pm 0.66^{\mathrm{a}}$ & $9.36 \pm 0.26^{\mathrm{a}}$ & $8.91 \pm 0.22^{\mathrm{a}}$ \\
\hline \multirow{2}{*}{ SNF yield (kg/day) } & Control & $2.93 \pm 0.09^{\mathrm{a}}$ & $2.99 \pm 0.12^{\mathrm{a}}$ & $3.15 \pm 0.09^{\mathrm{a}}$ & $3.02 \pm 0.07^{\mathrm{a}}$ \\
\hline & Treated & $3.14 \pm 0.19^{\mathrm{a}}$ & $2.78 \pm 0.28^{\mathrm{a}}$ & $3.09 \pm 0.23^{\mathrm{a}}$ & $3.05 \pm 0.17^{\mathrm{a}}$ \\
\hline \multicolumn{6}{|l|}{ Milk fat: } \\
\hline \multirow{2}{*}{ Fat $\%$} & Control & $2.25 \pm 0.29^{\mathrm{b}}$ & $3.36 \pm 0.44^{\mathrm{a}}$ & $3.91 \pm 0.41^{\mathrm{a}}$ & $3.17 \pm 0.24^{\mathrm{a}}$ \\
\hline & Treated & $3.56 \pm 0.32^{\mathrm{a}}$ & $2.72 \pm 0.43^{\mathrm{a}}$ & $4.90 \pm 0.40^{\mathrm{a}}$ & $3.82 \pm 0.20^{\mathrm{a}}$ \\
\hline \multirow{2}{*}{ Fat yield (kg/day) } & Control & $0.87 \pm 0.16^{\mathrm{b}}$ & $1.15 \pm 0.16^{\mathrm{a}}$ & $1.34 \pm 0.15^{\mathrm{a}}$ & $1.19 \pm 0.11^{\mathrm{a}}$ \\
\hline & Treated & $1.24 \pm 0.13^{\mathrm{a}}$ & $0.93 \pm 0.13^{\mathrm{a}}$ & $1.65 \pm 0.21^{\mathrm{a}}$ & $1.31 \pm 0.09^{\mathrm{a}}$ \\
\hline \multicolumn{6}{|l|}{ Milk sugar (Lactose): } \\
\hline \multirow{2}{*}{ Lactose $\%$} & Control & $4.74 \pm 0.06^{\mathrm{a}}$ & $4.76 \pm 0.11^{\mathrm{a}}$ & $5.13 \pm 0.15^{\mathrm{a}}$ & $4.88 \pm 0.08^{\mathrm{a}}$ \\
\hline & Treated & $4.78 \pm 0.18^{\mathrm{a}}$ & $4.46 \pm 0.36^{\mathrm{a}}$ & $4.94 \pm 0.18^{\mathrm{a}}$ & $4.72 \pm 0.16^{\mathrm{a}}$ \\
\hline \multirow{2}{*}{ Lactose yield (kg/day) } & Control & $1.61 \pm 0.05^{\mathrm{a}}$ & $1.63 \pm 0.07^{\mathrm{a}}$ & $1.72 \pm 0.05^{\mathrm{a}}$ & $1.65 \pm 0.04^{\mathrm{a}}$ \\
\hline & Treated & $1.67 \pm 0.12^{a}$ & $1.57 \pm 0.15^{\mathrm{a}}$ & $1.64 \pm 0.14^{\mathrm{a}}$ & $1.62 \pm 0.11^{\mathrm{a}}$ \\
\hline \multicolumn{6}{|l|}{ Milk protein: } \\
\hline \multirow{2}{*}{ Protein $\%$} & Control & $3.14 \pm 0.04^{\mathrm{a}}$ & $3.15 \pm 0.07^{\mathrm{a}}$ & $3.42 \pm 0.09^{\mathrm{a}}$ & $3.24 \pm 0.05^{\mathrm{a}}$ \\
\hline & Treated & $3.35 \pm 0.14^{\mathrm{a}}$ & $2.76 \pm 0.22^{\mathrm{b}}$ & $3.67 \pm 0.27^{\mathrm{a}}$ & $3.29 \pm 0.13^{\mathrm{a}}$ \\
\hline \multirow{2}{*}{ Protein yield (kg/day) } & Control & $1.06 \pm 0.03^{\mathrm{a}}$ & $1.08 \pm 0.04^{\mathrm{a}}$ & $1.14 \pm 0.03^{\mathrm{a}}$ & $1.09 \pm 0.02^{\mathrm{a}}$ \\
\hline & Treated & $1.16 \pm 0.07^{\mathrm{a}}$ & $1.02 \pm 0.11^{\mathrm{a}}$ & $1.23 \pm 0.13^{\mathrm{a}}$ & $1.13 \pm 0.07^{\mathrm{a}}$ \\
\hline \multicolumn{6}{|l|}{ Milk ash: } \\
\hline \multirow{2}{*}{ Ash $\%$} & Control & $0.66 \pm 0.01^{\mathrm{a}}$ & $0.67 \pm 0.02^{\mathrm{a}}$ & $0.73 \pm 0.02^{\mathrm{a}}$ & $0.69 \pm 0.01^{\mathrm{a}}$ \\
\hline & Treated & $0.68 \pm 0.02^{\mathrm{a}}$ & $0.62 \pm 0.05^{\mathrm{a}}$ & $0.73 \pm 0.01^{\mathrm{a}}$ & $0.68 \pm 0.02^{\mathrm{a}}$ \\
\hline \multirow{2}{*}{ Ash yield (kg/day) } & Control & $0.22 \pm 0.01^{\mathrm{a}}$ & $0.23 \pm 0.01^{\mathrm{a}}$ & $0.24 \pm 0.01^{\mathrm{a}}$ & $0.23 \pm 0.01^{\mathrm{a}}$ \\
\hline & Treated & $0.24 \pm 0.02^{\mathrm{a}}$ & $0.22 \pm 0.02^{\mathrm{a}}$ & $0.024 \pm 0.02^{\mathrm{a}}$ & $0.24 \pm 0.02^{\mathrm{a}}$ \\
\hline
\end{tabular}

Values are expressed as means \pm standard error (SE). Means within the same column of different litters are significantly different at $(\mathrm{P}<0.05)$.

\section{Milk-to-feed ratio:}

Probiotic supplementation in dairy cattle ration non significantly $(\mathrm{P} \geq 0.05)$ improved milk-to-feed ratio (table 7) during $1^{\text {st }}$ and $2^{\text {nd }}$ month postpartum by about $3.8 \%$ and $8.9 \%$ respectively, while reduced $(\mathrm{P} \geq 0.05)$ milk-to-feed ratio during $3^{\text {rd }}$ month of lactation by about $6.5 \%$ compared to control. Moreover, probiotic supplementation improved $(\mathrm{P} \geq 0.05)$ average milk-to-feed ratio throughout the whole experimental period by about $4.3 \%$ compared to control.

Table 7: Effect of dietary bacterial probiotic supplementation on postpartum milk-to-feed ratio of dairy cows.

\begin{tabular}{ccc}
\hline \multirow{2}{*}{ Stage of lactation (weeks postpartum) } & \multicolumn{2}{c}{ Treatment } \\
\cline { 2 - 3 } & Control & Probiotic supplementation \\
\hline $0-4$ & $1.82 \pm 0.09^{\mathrm{a}}$ & $1.89 \pm 0.11^{\mathrm{a}}$ \\
\hline $5-8$ & $1.69 \pm 0.07^{\mathrm{a}}$ & $1.84 \pm 0.15^{\mathrm{a}}$ \\
\hline $9-12$ & $1.38 \pm 0.04^{\mathrm{a}}$ & $1.29 \pm 0.10^{\mathrm{a}}$ \\
\hline $0-12$ (Average) & $1.62 \pm 0.06^{\mathrm{a}}$ & $1.69 \pm 0.11^{\mathrm{a}}$ \\
\hline
\end{tabular}

Values are expressed as means \pm standard error (SE). Means within the same row of different litters are significantly different at $(\mathrm{P}<0.05)$.

\section{Blood parameters}

Table (8) shows the means values of some blood serum units. It was observed that probiotic supplementation in dairy cattle ration had no significant effect on serum total protein, albumin,
AST and ALT concentrations compared to control. Moreover, probiotic non significantly $(\mathrm{P} \geq 0.05)$ increased serum triglycerides concentration by about $10.2 \%$ while, significantly increased serum cholesterol by about $62.3 \%$ compared with control. 
Table 8: Effect of dietary bacterial probiotic supplementation on some blood serum biochemical parameters of dairy cows.

\begin{tabular}{ccc}
\hline \multirow{2}{*}{ Items } & \multicolumn{2}{c}{ Treatment } \\
\cline { 2 - 3 } & Control & Probiotic supplementation \\
\hline Total protein $(\mathrm{g} / \mathrm{dl})$ & $10.99 \pm 0.78^{\mathrm{a}}$ & $9.59 \pm 0.41^{\mathrm{a}}$ \\
\hline Albumin $(\mathrm{g} / \mathrm{dl})$ & $3.27 \pm 0.46^{\mathrm{a}}$ & $3.54 \pm 0.23^{\mathrm{a}}$ \\
\hline Triglycerides $(\mathrm{mg} / \mathrm{dl})$ & $102.46 \pm 11.65^{\mathrm{a}}$ & $112.89 \pm 13.32^{\mathrm{a}}$ \\
\hline Total cholesterol $(\mathrm{mg} / \mathrm{dl})$ & $163.51 \pm 16.02^{\mathrm{b}}$ & $265.91 \pm 49.20 \mathrm{a}$ \\
\hline AST $(\mu / \mathrm{L})$ & $15.25 \pm 2.30^{\mathrm{a}}$ & $20.92 \pm 3.99^{\mathrm{a}}$ \\
\hline $\operatorname{ALT}(\mu / \mathrm{L})$ & $21.35 \pm 2.06^{\mathrm{a}}$ & $18.36 \pm 1.44^{\mathrm{a}}$
\end{tabular}

Values are expressed as means \pm standard error (SE). Means within the same row of different litters are significantly different at $(\mathrm{P}<0.05)$.

\section{Reproductive performance:}

Regarding reproductive performance of dairy cows (table 9), it was observed that days from calving to $1^{\text {st }}$ insemination was earlier by about 1.5 days, number of insemination per conception was less by about $4.4 \%$ and days open (DO) was shorter by about
9.5 days of cows in probiotic treated group compared to control. Moreover, probiotic supplementation in dairy cows ration improved conception rate $\%$ and repeat breeders $\%$ by about $21.9 \%$ and $55.6 \%$ respectively compared to control.

Table 9: Effect of dietary bacterial probiotic supplementation on reproductive performance of dairy cows.

\begin{tabular}{ccc}
\hline \multirow{2}{*}{ Items } & \multicolumn{2}{c}{ Treatment } \\
\cline { 2 - 3 } & Control & Probiotic supplementation \\
\hline Days from calving to $1^{\text {st }}$ insemination & $60.61 \pm 3.46 \mathrm{a}$ & $59.09 \pm 2.69 \mathrm{a}$ \\
\hline Insemination No. & $2.28 \pm 0.32 \mathrm{a}$ & $2.18 \pm 0.30 \mathrm{a}$ \\
\hline Days open & $93.06 \pm 7.57 \mathrm{a}$ & $83.56 \pm 8.88 \mathrm{a}$ \\
\hline Conception rate $(\%)$ at $1^{\text {st }}$ insemination & 27 & 27 \\
\hline Conception rate $(\%)$ at $2^{\text {nd }}$ insemination & 44 & 46 \\
\hline Conception rate $(\%)$ at $3^{\text {rd }}$ insemination & 11 & 27 \\
\hline Average conception rate $(\%)$ & 27.3 & 33.3 \\
\hline Repeat breeders $(\%)$ & 18.0 & 10.0
\end{tabular}

Values are expressed as means \pm standard error (SE). Means within the same row of different litters are significantly different at $(\mathrm{P}<0.05)$.

\section{Serum progesterone concentration:}

The obtained that indicated that feeding lactating cows on diets supplemented by bacterial probiotic non significantly $(\mathrm{P} \geq 0.05) \quad$ increased serum progesterone concentration (table 10) after both periods of $1^{\text {st }}$ inseminate and $2^{\text {nd }}$ insemination compared to control.

Table 10: Effect of dietary bacterial probiotic supplementation on serum progesterone during postpartum periods of dairy cows.

\begin{tabular}{cccc}
\hline \multirow{2}{*}{ Items } & $\begin{array}{c}\text { Days after } \\
\text { insemination }\end{array}$ & Control & Treatment \\
\cline { 2 - 4 } & $7-12$ & $1.43 \pm 0.08^{\mathrm{a}}$ & Probiotic supplementation \\
\hline $\begin{array}{c}\text { Progesterone }(\mathrm{ng} / \mathrm{ml}) \text { of } 1^{\text {st }} \\
\text { insemination }\end{array}$ & 20 & $1.35 \pm 0.38^{\mathrm{a}}$ & $1.62 \pm 0.24^{\mathrm{a}}$ \\
\hline $\begin{array}{c}\text { Progesterone }(\mathrm{ng} / \mathrm{ml}) \text { of } 2^{\text {nd }} \\
\text { insemination }\end{array}$ & $7-12$ & $0.55 \pm 0.30^{\mathrm{a}}$ & $0.58 \pm 0.26^{\mathrm{a}}$ \\
\cline { 2 - 4 } & 20 & $0.43 \pm 0.31^{\mathrm{a}}$ & $0.75 \pm 0.38^{\mathrm{a}}$ \\
\hline
\end{tabular}

Values are expressed as means \pm standard error (SE). Means within the same row of different litters are significantly different at $(\mathrm{P}<0.05)$.

\section{Postpartum uterine involution:}

Results presented in table (11) show that bacterial probiotic supplementation in dairy cows ration shorten the period required for postpartum uterine involution through reduction of uterine cervix, uterine right and left horn diameters during $3^{\text {rd }}$ to $4^{\text {th }}$ weeks postpartum by about $21.6 \%, 7.9 \%$ and $22.1 \%$ respectively compared to control. 
Table 11: Effect of dietary bacterial probiotic supplementation on postpartum uterine involution of dairy cows.

\begin{tabular}{|c|c|c|}
\hline \multirow{2}{*}{ Items } & \multicolumn{2}{|c|}{ Treatment } \\
\hline & Control & Probiotic supplementation \\
\hline \multicolumn{3}{|l|}{ Uterine cervix diameter/mm } \\
\hline At 22 - 29 days postpartum & $29.60 \pm 1.45^{\mathrm{a}}$ & $23.21 \pm 2.12^{b}$ \\
\hline At 38 - 45 days postpartum & $28.96 \pm 0.87^{\mathrm{a}}$ & $25.04 \pm 2.33^{\mathrm{a}}$ \\
\hline \multicolumn{3}{|c|}{ Uterine right horn diameter/mm } \\
\hline At 22 - 29 days postpartum & $24.60 \pm 2.15^{\mathrm{a}}$ & $22.65 \pm 2.07^{\mathrm{a}}$ \\
\hline At 38 - 45 days postpartum & $21.81 \pm 2.38^{\mathrm{a}}$ & $23.66 \pm 1.86^{\mathrm{a}}$ \\
\hline \multicolumn{3}{|c|}{ Uterine left horn diameter/mm } \\
\hline At 22 - 29 days postpartum & $26.43 \pm 2.44^{\mathrm{a}}$ & $20.58 \pm 1.74^{b}$ \\
\hline At 38 - 45 days postpartum & $20.77 \pm 2.72^{\mathrm{a}}$ & $20.89 \pm 2.68^{\mathrm{a}}$ \\
\hline
\end{tabular}

Values are expressed as means \pm standard error (SE). Means within the same row of different litters are significantly different at $(\mathrm{P}<0.05)$.

\section{DISCUSSION}

\section{Body condition score}

Results revealed that cows in the treated group lost less BCS till day $4^{\text {th }}$ weeks postpartum than control then they gained more score till $3^{\text {rd }}$ month postpartum than control did, These results indicate that treated cows had better transition period and lower negative energy balance during the transition period which can be explained with Nocek et al. (2003) who showed that during the transition period cows receiving DFM had a higher concentration of blood glucose and insulin and a lower concentration of both $\beta$ hydoxybutyrate (BHBA) and non-esterified fatty acids (NEFA) when compared with the control cows. And agree with another study (Peng et al., 2012) who reported lower plasma NEFA after calving with cows that were fed Bacillus subtilisnatto. Lower concentration of NEFA indicates that cows are mobilizing less energy from adipose deposits to meet their high energy requirements. The decrease in BHBA levels indicate more efficient use of nutrients for production and a decrease in negative energy balance (Drackley, 1999). Also Luan et al. (2015) observed less subclinical ketosis after calving with cows consuming Bacillus pumilus as a DFM. Less ketones and ketosis suggest that supplementing probiotics can decrease the amount of energy that cows take from adipose tissue. When blood glucose is made available and cows are mobilizing less fatty acids from adipose tissues, glucose can route to the mammary gland to produce more milk.

\section{Milk production and composition:}

Response of average lactation performance $(+7.3 \%)$ throughout the whole experimental period in bacterial probiotics treated group compared to control is of large biological significance and would result in favorable ratio of extra income to probiotic cost.
Modern dairy farms are targeting high milk production utilizing feed composed of high concentrates to meet the metabolic demand of the higher milk production. Such feeding system is associated with metabolic dysfunction like rumen acidosis especially during poor feeding condition and composition. Therefore, probiotics are suggested as an effective mechanism of preventing or treating ruminal acidosis and/or improve animals' performances (Lettat et al., 2012). Thus, according to Nocek and Kautz (2006) the supplementation of probiotics (i.e. Yeast and Enterococcos mix, Propionibacterium (strain-P169), to dairy cows have significantly improved milk yield in comparison to control. Also, Yasuda et al. (2007) reported 3-16\% increase in milk production in dairy cows by supplementing the diet with probiotics. Peng et al. (2012) who reported an even greater increase in milk yield of 3.1 and $3.2 \mathrm{~kg} / \mathrm{d}$ with Bacillus subtilisnatto.

Like milk yield, the effects of probiotics in milk composition were also inconsistent across different literatures. Consequently (Vibhute et al., 2011; Rossow, 2018) were reported significant improvement in milk fat \% after supplementation of probiotics. In contrary, Dutta and Kundu, 2008. In similar ways, different authors reported that probiotic supplementation have significantly affected milk protein (\%) (Rossow et al., 2018; Hossain et al., 2014; Yalcin et al., 2011; Vibhute et al., 2011), milk lactose (\%) (Nocek and Kautz, 2006; Hossain et al., 2014) and SNF\% (Hossain et al., 2014). While, some authors have showed that, there was no significant influence of probiotics on yield of milk protein $\%$ (Nocek and Kautz, 2006; Weiss et al., 2008; Maamouri et al., 2014), lactose \% (Weiss et al., 2008; Maamouri et al., 2014; Stella et al., 2007) and Ashe \% (Maamouri et al., 2014). 


\section{Dry matter intake and milk-to-feed ratio:}

Bacterial probiotic supplementation numerically improved DM intake and milk-to-feed ratio during postpartum period compared with control. Supports these findings Qiao et al. (2010) and Souza et al. (2017) observed no response to Bacillus subtilis on intake of lactating cows over a 10-weekperiod. In addition, similar results were reported on lactating buffaloes fed Biovet as micro-organisms added to their diets (Gujjar et al., 2006) or dairy goats fed Lecture as a bacterial feed additive (Ahmed et al., 2008). However, Aikman et al. (2008) observed no difference in DM intake between the control and treated cows fed two TMR's differing in level of concentrate and supplemented with direct-fed microbial (DFM) during the first 14 weeks of lactation.

\section{Biochemical Blood parameters:}

Blood biochemical parameters are commonly used to assess the nutritional and physiological status of lactating animals (Kafilzadeh et al., 2019). Results declared that treated group had a higher cholesterol and AST concentrations than control even they are within normal range, In agreement with these results, Mousa et al. (2012) observed significantly higher AST activity, without changes on ALT activity, in the blood plasma of dairy ewes fed probiotics compared with the controls. The AST activity may bedue to increased metabolism of amino acids, as energy source or glucose precursors (Caldeira et al., 1999), whereas increased cholesterol levels would indicate a greater absorption of nutrients in the intestines (Chiofalo et al., 2004).

\section{Reproductive performance and serum progesterone:}

These results indicated that feeding lactating cows on diets supplemented with bacterial probiotics had beneficial effects on reproductive performance of lactating cows as compared control. It is of interest to note that dietary supplementation of bacterial probiotics markedly increased concentration of progesterone in blood serum of cows after $1^{\text {st }}$ and $2^{\text {nd }}$ inseminations periods as compared to control group, but the differences were not significant (table 10). Result agreed with Mostafa et al. (2014) who reported that dietary supplementation of probiotics improved productive and reproductive performances of lactating cows. Such finding might be attributed either to stimulation of the immune system (Rodrigues et al., 2000; Young, 2012) or improvement of the health condition of the supplemented cows by probiotics (Kalmus et al., 2009).

\section{Postpartum uterine involution:}

The restoration of the uterus to its normal nonpregnant size and function after parturition is termed uterine involution which depends on the rate of myometrial contractions, elimination of bacterial infection and the histological regeneration of the endometrium (Elmetwally et al., 2016). Involution of the uterus is necessary before the cow can conceive again. As a result of the removal of the fetus, oxytocin and prostaglandin F2 $\alpha$ (PGF2 $\alpha$ ) production reduced the uterine size (Edquist et al., 1978). The reduction of uterine cervix and horn diameters early of dairy cows fed on probiotic supplemented diet compared to control reflected to better fertility and conception rate. Improvement of uterine involution may be to higher serum progesterone concentration in dairy cows fed on probiotic supplemented diet compared to control. The present data are supported by (Sheldon et al., 2003) indicated that prostaglandins have a role in controlling uterine involution.

\section{CONCLUSION}

Using the produced probiotics supplement in dairy cows ration improved cows health and improved its transition period, BCS, milk production and milk quality. It also increased cows' energy utilization and improved reproductive performance and post-partum uterine involution. Also using probiotics generally did not adversely affect the levels of some biochemical indices of treated cows.

\section{REFERENCES}

Abu-El-Ella, A.A. and Kommonna, O.F. (2013): Reproductive performance and blood constituents of Damascus goats as affected by yeast culture supplementation. Egyptian J. Sheep Goat Sci. 8 (1): 171-187.

Aikman, P.C.; Henning, P.H.; Jones, A.K.; Potteron, S. and Siviter, J. (2008): Effect of administration of Megasphaeraelsdenii NCIMB 41125 lactate utilizing bacteria in early lactation on the production, health and rumen environment of highly productive dairy cows fed a high concentrate diet. KK Animal Nutrition Internal Report, KKAN, Umbogintwini, South Africa.

Ahmed, M.E; Shehata, E.I.; Abou Ammou, F.F.; abdel-Gowad, A.M. and Aid, K.M. (2008): Milk production,feedconvertion rate and reprodution of Zaraibi goat in response to bacterial feed additive during late pregnancy and lactation Egyption. Journal of animal production,45: 189-203.

AlZahal, O.; McGill, H.; Kleinberg, A.; Holliday, J.I.; Hindrichsen, I.K.; Duffield, T.F. and McBride, B.W. (2014): Use of a direct-fed microbial product as a supplement during the transition period in dairy cattle. J. Dairy Sci. 97: 7102-7114.

AOAC International (2000): Official. 17th Methods of Analysis ed. AOAC Int., Arlington, VA. 
Autrere, M.B. and Benson, H. (1976): Progesterone: An overview and recent advances, J. Par. Sci.; 65: 783-800.

Biggs, D.A.; Johnsson, G. and Sjaunja, L.O. (1987): Analysis of fat, protein, lactose and total solids by infra-red absorption. IDF Bull. 208: 22-29.

Caldeira, RM.; Almeida, MA.; Santos, CC.; Vasques, MI. and Portugal, AV. (1999): Daily variation in blood enzymes and metabolites in ewes under three levels of feed intake. Can J Anim Sci. 79: 157-164.

Cannon, T.W.; Dye, J.E. and Toutenhoofd, V. (1974): The mechanism of precipitation formation in northeastern Colorado cumulus II. Sailplane measurements. J. Atmos. Sci., 31, 2148-2151, doi

Can West, DHI and Valacta (2013): Culling and replacement rates of dairy herds in Canada. Accessed Jun. 23, 2014.http:// www.dairyinfo.gc.ca/pdf/genetics-cull_e.pdf

Chiofalo, V.; Liotta, L. and Chiofalo, B. (2004): Effects of the administration of Lactobacilli on body growth and on the metabolic profile in growing Maltese goat kids. Reprod Nutr Dev. 44: 449-457.

Chávarri, M.; Marañón, I.; Ares, R.; báñez, F.C.; Marzo, F. and Villarán, M. del Carmen (2010): Microencapsulation of a probiotic and prebiotic in alginate-chitosan capsules improves survival in simulated gastrointestinal conditions. International Journal of Food Microbiology 142 (2010) 185-189.

Doumas, B.T.; Watson, W.A. and Briggs, H.G. (1971): Albumin standards and the measurement of serum albumin with bromocresol green. Clin. Chem. Acta 31: 87-97.

Drakely, K. (1999): Biology of dairy cows during the transition period: the final frontier? J. Dairy Sci. 82: 2259-2273.

Dutta, TK. and Kundu, SS. (2008): Response of mixed viable probiotics culture on milk production and nutrient availability in crossbred mid lactating cows. Indian Journal of Animal Sciences. 78(5): 531-535

Edquist, L.E.; Lindell, J.O. and Kindahl, H. (1978): Proc. 9th Int. Anim. Reprod. Artific. Insem., Madrid.

Elmetwally, M.A.; Montaser, A.; Elsadany, N.; Bedir, W.; Hussein, M. and Zaabel, S. (2016): Effects of Parity on Postpartum Fertility Parameters in Holstein Dairy Cows. IOSR Journal of Agriculture and Veterinary Science, 9, 91-99.

Ferguson, J.D. (2001): Nutrition and reproduction in dairy herds. In: Proc. 2001 Intermountain Nutr. Conf., Salt Lake City, UT, pages 65-82

Fuller, R. (1989): Probiotics in man and animals. J. Appl. Bacterial. 66: 365-378.

Gujjar, S.R.; Ahmed, M. and Javid, R.S. (2006): Effect of biovet and probitic (BM-
Technology) on milk production of lactating buffaloes livestock production research institute, Bahadurnagar, Okara, Pakistan Pak Vet J, 2006, 26(4): 201-203

Hossain, FMA.; Islam, MM.; Aral, A. and Iliyas, N. (2014): Supplementing probiotics in multiparous crossbred cows ration provoke milk yield and composition. Online J. Anim. Feed Res. 4(2): 18-24

Kafilzadeh, F.; Payandeh, S.; Gómez-Cortés, P.; Ghadimi, D.; Schiavone A. and Andrés L. Martínez Marín (2019): Effects of probiotic supplementation on milk production, blood metabolite profile and enzyme activities of ewes during lactation, Italian Journal of Animal Science, 18:1, 134-139, DOI: 10.1080/1828051X.2018.1496040

Kalmus, P.; Orro, T.; Waldmann, A.; Lindjarv, R. and Kask, K. (2009): Effect of yeast culture 49 on milk production and metabolic and reproductive performance of early lactation dairy cows. Acta. Vet. Scand. 51: 31-38.

Kritas, S.K.; Govaris, A.; Christodoulopoulos, G. and Burriel, A.R. (2006): Effect of Bacillus licheniformis and Bacillus subtilis supplementation of ewe's feed on sheep milk production and young lamb mortality. J. Vet. Med. Series A 53: 170-173.

Lettat, A.; Nozière, P.; Silberberg, M.; Morgavi, DP.; Berger, C. and Martin, C. (2012): Rumen microbial and fermentation characteristics are affected differently by bacterial probiotic supplementation during induced lactic and subacute acidosis in sheep. BMC Microbiology.; 12(1): 142.

Luan, S.; Duersteler, M.; Galbraith, E.A. and Cardoso, F.C. (2015): Effects of direct-fed Bacillus pumilus $8 \mathrm{G}-134$ on feed intake, milk yield, milk composition, feed conversion, and health condition of pre- and postpartum Holstein cows. J. Dairy Sci. 98: 6423-6432.

Maamouri, O.; Selmi, H. and M'hamdi, N. (2014): Effects of yeast feed supplement on milk production and its composition in Tunisian Holstein Friesian cows. Scientiaagriculturaebohemica. 45(3): 170-174.

Mongkolthanaruk, W. (2012): Classification of Bacillus beneficial substances related to plants, humans, and animals. J. Microbiol. Biotechnol. 22: 1597-1604.

Mousa, K.M.; El-Malky, O.M.; Komonna, O.F., S.E. Rashwan, S.F. (2012): Effect of some yeast and minerals on the productive and reproductive performance in ruminants. J. Am. Sci. 8 (2): 291-303.

Mostafa, T.H.; Elsayed, F.A.; Ahmed, M.A. and Elkholany, M.A. (2014): Effect of using some feed additives (two- probiotics) in dairy cow rations on production and reproductive performance Animal Production Research Institute, Agriculture Research Center, 
Ministry of Agriculture, Egypt Egyptian J. Anim. Prod. (2014) 51(1): 1-11

Nocek, J. and Kautz, W. (2006): Direct-fed microbial supplementation on ruminal digestion, health, and performance of pre-and postpartum dairy cattle. J. Dairy Science, 89(1): 260-266.

Nocek, J.E.; Kautz, W.P.; Leedle, J.A.Z. and Block, E. (2003): Direct-fed microbial supplementation on the performance of dairy cattle during the transition period. J. Dairy Sci. 86: 331-335.

Peng, H.; Wang, J.Q.; Kang, H.Y.; Dong, S.H.; Sun, P.; Bu, D.P. and Zhou, L.Y. (2012): Effect of feeding Bacillus subtilisnatto fermentation product on milk production and composition, blood metabolites and rumen fermentation in early lactation dairy cows. J. Anim. Physiol. Anim. Nutr. 96: 506-512.

Qiao, G.H; Shan, A.S.; Ma, N.; Ma, Q.Q. and Sun, Z.W. (2010): Effect of supplemental Bacillus cultures on rumen fermentation and milk yield in Chinese Holstein cows. J. Anim. Physiol. Anim. Nutr. 94: 429-436.

Rodrigues, A.C.; Cara, D.C.; Fretez, S.H.; Cunha, F.Q.; Vieira, E.C.; Nicoli, J.R. and Vieira, L.Q. (2000): Saccharomyces boulardii stimulates $\operatorname{IgA}$ production and the phagocytic system of gnotobiotic mice. J. Appl. Microbiol. 89: 404-414.

Rossow, HA.; Riordan, T. and Riordan, A. (2018): Effects of addition of a live yeast product on dairy cattle performance. Journal of Applied Animal Research, 46(1): 159-163.

Stein, G. and Athans, M. (1987): The LQR/LTR Procedure for Multivariable Feedback Control Design IEEE Transactions on Automatic Control, AC-32 (2) pp. 105-113

Sheldon, I.H.; Noakes, D.E.; Rycroft, A.N. and Dobson, H. (2003): The effect of intrauterine administration of estradiol on postpartum uterine involution in cattle.Theriogenology, 59: 1357.

SPSS (2007): Statistical program for social science, Version.

Souza, R.A.*R.J.; Tempelman, M.S.; Allen, W.P.; Weiss, J.K.; Bernard, M.J. and Vande Haar, 1. (2017): Predicting nutrient digestibility in high-producing dairy cows Volume 101, Issue 2, Pages 1123-1135

Stella, AV.; Paratte, R.; Valnegri, L.; Cigalino, G.; Soncini, G. and Chevaux, E. (2007): Effect of administration of live Saccharomyces cerevisiae on milk production, milk composition, blood metabolites and fecal flora in early lactating dairy goats. Small Rumin Res. 67: 7-13.
Surinder, S. Bhalaru; Tiwana, M.S. and Narinder Singh (1987): Effect of body condition at calving on subsequent reproductive performance in buffaloes. Indian J. Anim. Sci. 57: 33.

Timmerman, H.M.; Koning, C.J.; Mulder, L.; Rombouts, F.M. and Beynen, A.C. (2004): Monostrain, multistrain and multispecies probiotics - A comparison of functionality and efficacy. Int. J. Food Microbiol. 96, 219-233.

Van Soest, P.V.; Robertson, J.B. and Lewis, B.A. (1991): Methods for dietary fiber, neutral detergent fiber, and nonstarch polysaccharides in relation to animal nutrition.Journal of dairy science, 74(10), 3583-3597.

Vibhute, VM.; Shelke, RR.; Chavan, SD. and Nage, $S P$. (2011): Effect of probiotics supplementation on the performance of lactating crossbred cows. Vet. World. 4(12): 557-561

Westwood, C.T.; Lean, I.J.; Garvin, J.K. and Wynn, $P$. (2000): Effects of genetic merit and varying dietary protein degradability on lactating dairy cowsJ. Dairy Sci., 83 (2000), pp. 2926-2940

Walter, J. (2005): The microecology of lactobacilli in the gastrointestinal tract. In: Tannock, G. (Ed.), Probiotics and Prebiotics, Scientific Aspects. Caister Academic Press, UK.

Weiss, W.; Wyatt, D. and McKelvey, T. (2008): Effect of feeding propionibacteria on milk production by early lactation dairy cows. Journal of Dairy Science. 91(2): 646-652.

Westwood, C.T.; Lean, I.J.; Garvin, J.K. and Wynn, $P$. (2000): Effects of genetic merit and varying dietary protein degradability on lactating dairy cows. J. Dairy Sci. 83: 2926-2940.

Yalcin, S.; Can, P.; Gurdal, AO.; Bagc, B. and Eltan, $O$. (2011): The nutritive value of live yeast culture and its effect on milk yield, milk composition and some blood parameters of dairy cows. Asian-Aust. J. Anim. Sci., 24(10): 1377- 1385 .

Yasuda, K.S.S.; Hashikawa, Y.; Tomita, S. Shibala and Tsuneo, F. (2007): "A new symbiotic consisting of lactobacillus caseicasei and dextran improves milk production of Holstein dairy cows," Journal of Veterinary Medical Science, vol. 69, no. 2, pp. 205, 2007

Young, DS. (1990): Effects of drugs on clinical laboratory tests. Third edition.3: 6-12.

Young, R.T. (2012): Yeast cell wall supplementation alters the performance and health of newly received crossbred heifers. Thesis Master Degree of Science. Graduate Fact 


\section{تقييم تاثير استخدام محفزات نمو بكتيرية محلية الاتتاج علي الكفاعة الانتاجية والتناسلية في أبقار الهولثتين الحلابة

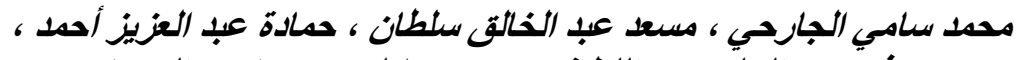

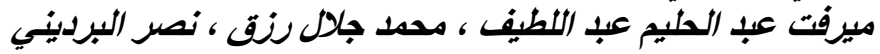

E-mail: m_elgarhi2005@yahoo.com

Assiut University web-site: www.aun.edu.eg

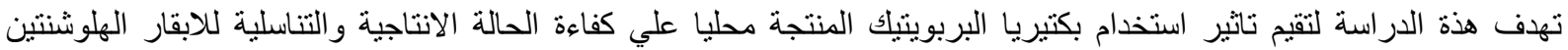

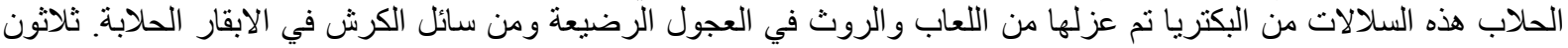

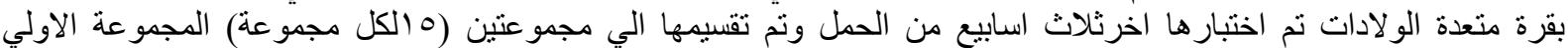

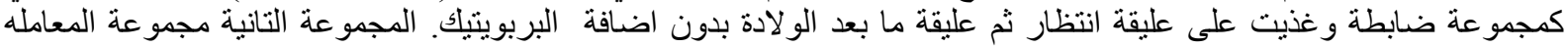

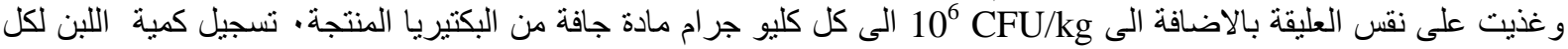

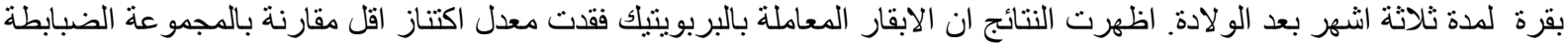

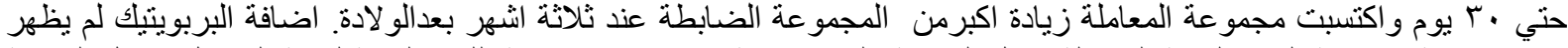

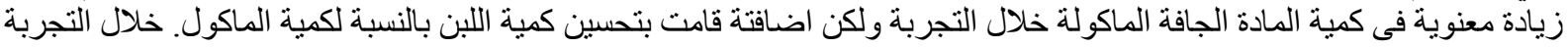

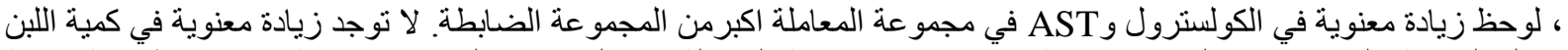

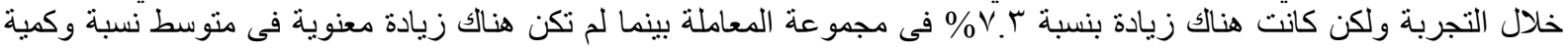

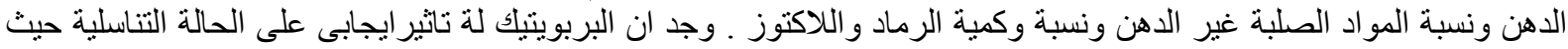

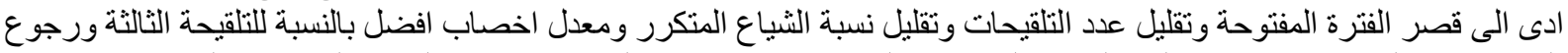

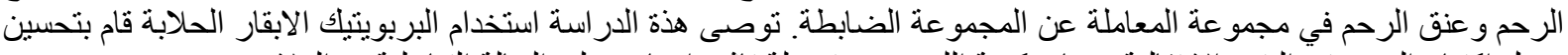

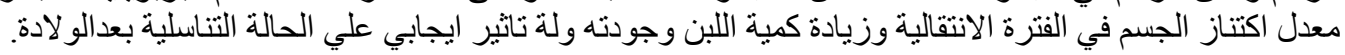

الكلمات الدالة: بكتيرياالبربويتيك ، كمية اللبن ، جودة البن و الكفاءة التناسلية. 